\title{
High Fidelity Imaging of Earth Materials by LA- ICP-TOF-MS
}

\author{
J.M.COTTLE ${ }^{1}$, A.R.C. KYLANDER-CLARK ${ }^{1}$, C.
}

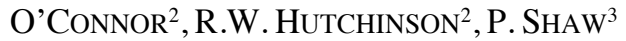

${ }^{1}$ Department of Earth Science, University California, Santa Barbara, CA 93106, USA (*correspondence: jcottle@ucsb.edu)

${ }^{2}$ Elemental Scientific Lasers, Bozeman, MT, USA (coconnor@icpms.com, rhutchinson@icpms.com)

${ }^{3} \mathrm{Nu}$ Instruments, Unit \&4 Clywedog Road South, Wrexham, LL13 9XS, UK

Recent advances in laser ablation systems and mass spectrometers have enabled high-speed acqusition of high spatial resolution 2-D and 3-D 'images' of elemental and isotopic variations in a range of materials. With some notable exceptions, the majority of applications of this potentially transformative technique have been in the field of biological imaging, and have not as yet found widespread application in the field of earth science. Here we combine two recently developed technological advances to demonstrate the ability of this technique to resolve micron-scale elemental and isotopic zoning in a range of earth materials. We specifically target accessory minerals (e.g., zircon, monazite, titanite), rock forming minerals (e.g., garnet, biotite) and carbonates (e.g., foraminifera, stalagmites) to assess the potential applications of this method. Analytical instrumentation consists of a newly developed "TwoVol3" laser ablation chamber installed on a NWR193 laser ablation system (Elemental Scientific Lasers) connected to a "Vitesse" timeof-flight inductively coupled plasma mass spectrometer $(\mathrm{Nu}$ Instruments). In Laser-based imaging applications, there is a key balance between the speed of acqusition and the required spatial resolution that places practical limits on the area that can be mapped and/or the spatial resolution with which differences in elemental concentrations can be detected. We present data demonstrating that the ultrafast washout ( $1 \mathrm{~ms})$, nm-scale stage precision, and the across-chamber $<1 \%$ elemental reproducibility of the "TwoVol3" cell coupled with the ultrafast acquisition rate of the "Vitesse" increases the limits by which high-resolution, multi-element/isotopic maps can be generated in a given period of time. Together, these instrument attributes enable routine production of multielement images at a rate of up to 1000 pixels per second at detection limits in the ppm-range. 